\title{
Simulation and visualization of fundamental optics phenomenon by LabVIEW
}

Bohan Lyu

Bohan Lyu, "Simulation and visualization of fundamental optics phenomenon by LabVIEW," Proc. SPIE 10452, 14th Conference on Education and Training in Optics and Photonics: ETOP 2017, 104523R (16 August 2017); doi: 10.1117/12.2257794

SPIE Event: 14th Conference on Education and Training in Optics and Photonics, ETOP 2017, 2017, Hangzhou, China 


\title{
Simulation and Visualization of Fundamental Optics Phenomenon by LabVIEW
}

\author{
Bohan Lyu \\ Bohan Photonics, 8F-2, No.125, Ln. 165, Yong-an Rd., Yongkang Dist., Tainan City 710, Taiwan
}

\begin{abstract}
Most instructors teach complex phenomenon by equation and static illustration without interactive multimedia. Students usually memorize phenomenon by taking note. However, only note or complex formula can not make user visualize the phenomenon of the photonics system. LabVIEW is a good tool for in automatic measurement. However, the simplicity of coding in LabVIEW makes it not only suit for automatic measurement, but also suitable for simulation and visualization of fundamental optics phenomenon. In this paper, five simple optics phenomenon will be discuss and simulation with LabVIEW. They are Snell's Law, Hermite-Gaussian beam transverse mode, square and circular aperture diffraction, polarization wave and Poincare sphere, and finally Fabry-Perrot etalon in spectrum domain.
\end{abstract}

Keywords: Simulation, Optics Education, Photonics Education, LabVIEW, Visualization

\section{INTRODUCTION}

The education in physics and photonics does not change much in the past 20 years. Is always an instructor in front the blackboard, and derive the complex formula. Students take some notes and try their best to understand the meaning of the formula. Some instructors are good at drawing, and they can draw refractive index ellipsoid, Poincare sphere, the moving or electric and magnetic wave on the blackboard. Some students are not good at drawing, and they can not fully copy what they see on the blackboard. The lost the chance to understand the optics theory. The UNESCO programe"Active Learning in Optics and Photonics" (ALOP) urge instructor should make several inexpensive experiment and interactive ways to help student understand the conceptual idea in photonics [1].

Now more and more instructors began to put the software demonstration in teaching. For example, use software to demonstrate propagation of electromagnetic waves through homogeneous media [2]. Using python to demonstrate diffraction phenomenon [3], or commercial software to do ray tracing optics [4] is the trend for teaching. Traditionally, LabVIEW is use for experiment automation, and several good instructor use it create very interesting experiment, such teaching Fresnel incoherent correlation holography [5] or undergraduate photonics experiment about computer controlled instrumentation and data acquisition [6]. Those are very good example in photonics laboratory. Nevertheless, LabVIEW has very powerful user interface design ability. With a short time of learning, instructor can make 3D demonstration of polarization wave or intensity plot of the diffraction pattern.

\section{TEACHING WITH LABVIEW VISUALIZATION}

Many optics laboratories already use National Instrument LabVIEW as experimental automation software, because its graphic interface make user who is not software background can easy start their creation. Typically, LabVIEW work bench has two parts. One is front panel and the other is block diagram. Block diagram is the coding place where define the logical function or algorithm of the program. The front panel is for operation the program or user changes parameters when running the program. LabVIEW has extraordinary plot function and easily to setup user interface. It is not only useful for laboratories automatic measurement, but also suitable for use demonstrate optics phenomenon. It is very easy to build track bar or numeric input. Visualization tool such as xy-plot, 2D picture plot, intensity plot, 3D plot are also ready for use.

14th Conference on Education and Training in Optics and Photonics: ETOP 2017, edited by Xu Liu,

Xi-Cheng Zhang, Proc. of SPIE Vol. 10452, 104523R - (c) 2017 ICO, IEEE, OSA, SPIE

CCC code: $0277-786 \mathrm{X} / 17 / \$ 18 \cdot$ doi: $10.1117 / 12.2257794$

Proc. of SPIE Vol. 10452 104523R-1 
Optical science uses much mathematics to describe the electric and magnetic wave interact with the substance. Use software visualization has at least three merits. First, the human eyes can not recognize the polarization of the light. It is very difficult to share the idea of polarization state to students. It always depends on students' imagination to figure out what is going on. However, polarization state is a very important characteristic in liquid crystal display (LCD). Liquid crystal changes the polarization from one polarization to another polarization. With polarizer and analyzer pairs, LCD becomes an optical valve to allow or stop the light. Besides, the relationship between polarization state and Poincare sphere sometimes confuse student. Thus, the first advantage of software visualization is making invisible phenomenon visible. Second, software can also help simulation the experiment. For example, Snell's law describes the relationship about the incident light angle, input refractive index, transmitted light. Fabry Perrot Etalon describes the transmittance in different etalon gap and surface reflectance. For real experiment, it is possible the change the incident angle, but very hard to change the refraction index instantly. As in Fabry Perot etalon, it is impossible to change the reflectance instantly when measuring the transmittance spectrum. Hence, the second advantage is help student do virtual experiment. Finally, student is familiar with optical simulation parameters also help student build their own instrument in the future. The technology improves very rapid and some parameters of the sample can not measurement directly such as cell gap or phase retardation of the liquid crystal cell. The instrument need very sophisticated physical model to fitting the answer. Building simulation can also improve the student's ability to compare the experiment and simulation. Before going to the laboratories, one can use simulation to do predict the experiment result. The future engineer or scientist can apply the concept of computer-aid-design (CAD) in early stage learning.

With software visualization, student can change the parameters and see the output result. After repeated operation student can build up more physical sense about the phenomenon not only equation.

\section{OPTICS SIMULATION WITH LABVIEW}

\subsection{Snell's law}

Snell's law is the fundamental formula describes the relationship among incident angle, refractive angle, refractive index of medium. In this program, user can drag the incident angle $\theta_{1}$ track bar and the program will auto compute the refractive angle $\theta_{2}$. In the Figure 1, refraction and total internal reflection are demonstrated. The refractive index in medium 1 and medium 2 are $\mathrm{n}_{1}$ and $\mathrm{n}_{2}$, respectively. The Snell's law is shown in the eq (1). User can change the parameters and observe what will change after the manipulation. Total internal refraction can also observe when the light incident from high refractive index medium to low refractive index medium.

$$
\mathrm{n}_{1} \sin \theta_{1}=\mathrm{n}_{2} \sin \theta_{2}
$$

(1)

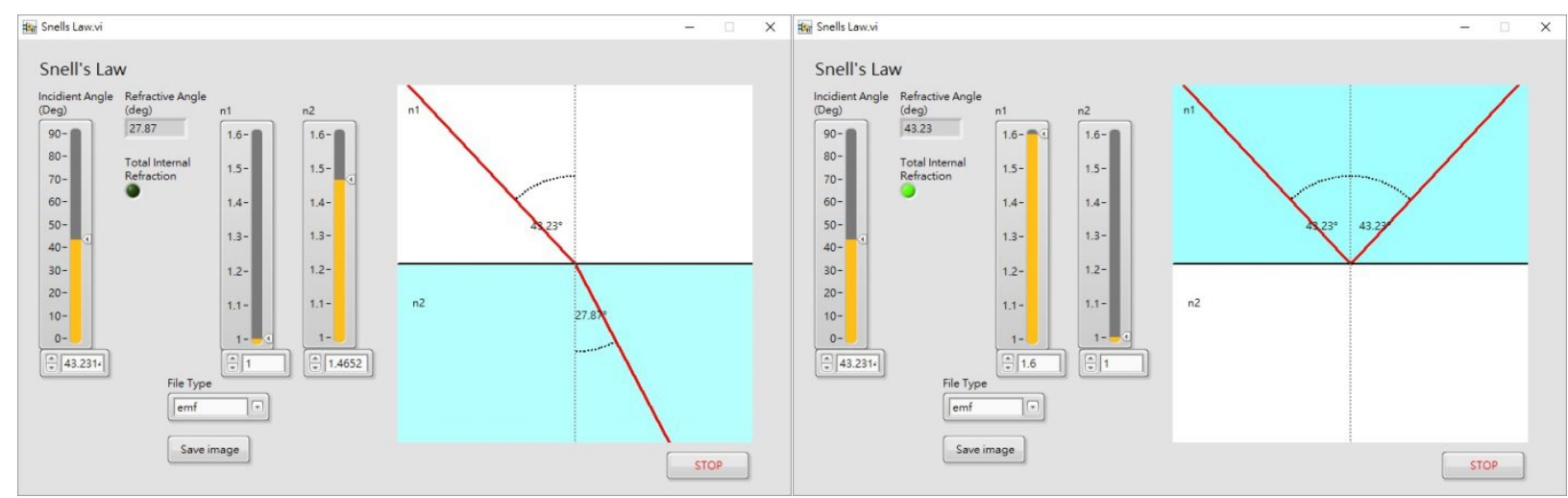

(a)

(b)

Figure 1 (a) Illustration of Snell's Law from low refractive index medium to high refractive index medium. (b) Illustration of Snell's Law from high refractive index medium to low refractive index medium. The incident angle is larger than critical angle, and therefore it lay in the total internal reflection (TIR) region. http://dx.doi.org/10.1117/12.2257794.1 


\subsection{Hermite-Gaussian beam transverse mode}

The Gaussian beam is a solution of paraxial Helmholtz equation. It can also decompose the solution in many orthogonal higher order forms. Hermite-Gaussian is a series solution of this equation in Cartesian coordinates [8]. The electric field of the solution is shown in eq (2) and (3). $\omega(\mathrm{z})$ is the spot size in $z ; \omega_{0}$ is the spot size when $z=0 \lambda$ is the wavelength of light; $\mathrm{R}(\mathrm{z})$ is the curvature in $\mathrm{z} . \mathrm{H}_{1}$ is the Hermite polynomial of order l.n is the refractive index of medium. An alternate orthogonal higher order solution in cylindrical coordinates is so-called Laguerre-Gaussian beam. The complexity of the Hermite-Gaussian beam traverse mode make student hard to realize how the light is look like.

$$
\begin{gathered}
E_{1, \mathrm{~m}}(x, y, z)=E_{0} \frac{\omega_{0}}{\omega(z)} H_{l}\left(\sqrt{2} \frac{x}{\omega(z)}\right) H_{m}\left(\sqrt{2} \frac{x}{\omega(z)}\right) \times \exp \left(-\frac{x^{2}+y^{2}}{\omega^{2}(z)}-i k \frac{x^{2}+y^{2}}{2 R(z)}-i k z+i(l+m+1) \eta\right) \\
\text { Where } \eta(\mathrm{z})=\tan ^{-1}\left(\frac{\lambda z}{\pi \omega_{0}^{2} n}\right)
\end{gathered}
$$

In this program, user can select the order of the Hermite-Gaussian beam and drag 3D plot for viewing the laser beam from different view direction. In the Figure 2, there are TEM00 and TEM11, the left part is the intensity distribution and the right part is the 3D plot of the Hermite-Gaussian beam. By visualization of the intensity distribution the, user cultivate more sense of Hermite-Gaussian beam.

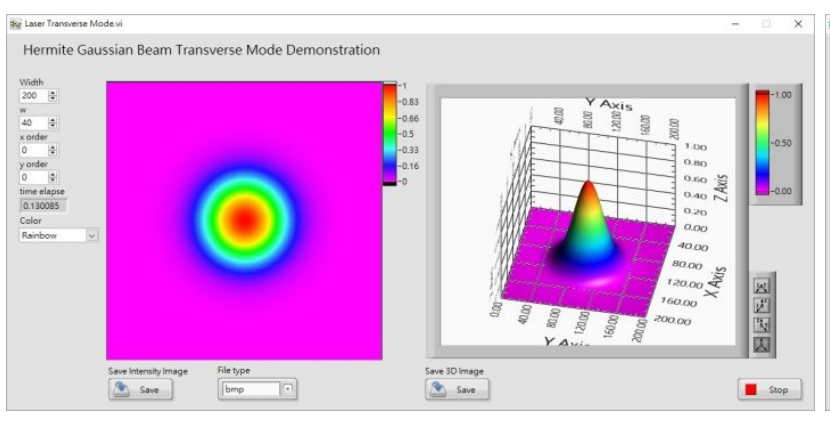

(a)

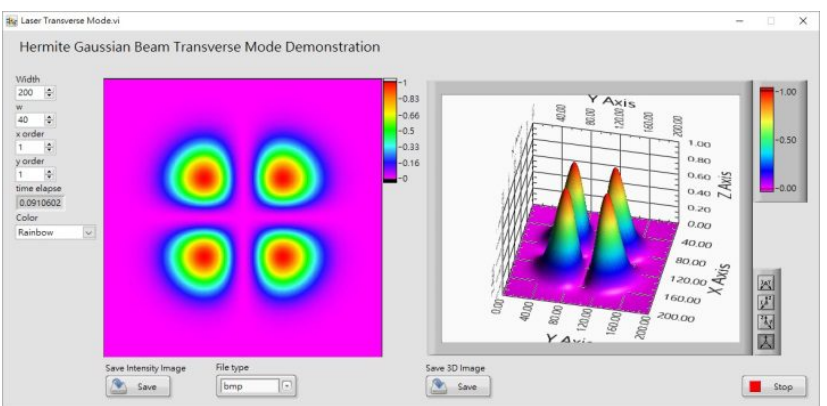

(b)

Figure 2 (a) Fundamental mode of Hermite-Gaussian beam which is TEM00 (b) TEM11 mode of Hermite-Gaussian beam. http://dx.doi.org/10.1117/12.2257794.2

\subsection{Square and circular aperture diffraction}

Diffraction of the light shows the wave property of the light. People think light is only a particle for a long time in history. Therefore, diffraction is a proof to show the light has the wave property. Usually after a long discussion in class, the textbook will jump out an equation to describe the phenomenon. That is Fresnel diffraction [9], as show in eq (4). In the beginning, user can learn two kinds of aperture and see how the aperture size affects the diffraction pattern. The smaller aperture size the larger diffraction angle. The distance between 0 order and 1 order will be wider. In the

Figure 3, square and circular apertures are demonstrated. The left part is the aperture and the right part is diffraction. By visualization of the diffraction pattern, student can learn this phenomenon in classroom. In the future, the simulation can use different shapes of aperture or different phase plate. Student can practice the computer generated hologram phase mask, to see how hologram is work.

$$
E(x, y, z)=\frac{e^{i k z}}{i \lambda z} \iint_{-\infty}^{+\infty} E\left(x^{\prime}, y^{\prime}, 0\right) e^{\frac{i k}{2 z}\left[\left(x-x^{\prime}\right)^{2}+\left(y-y^{\prime}\right)^{2}\right]} d x^{\prime} d y^{\prime}
$$




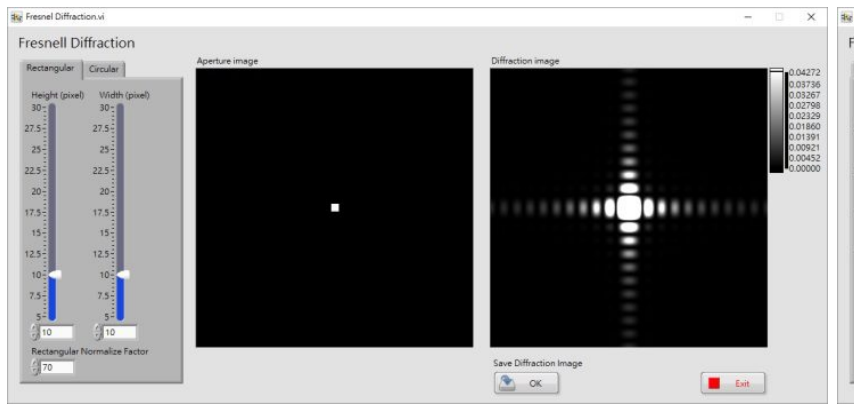

(a)

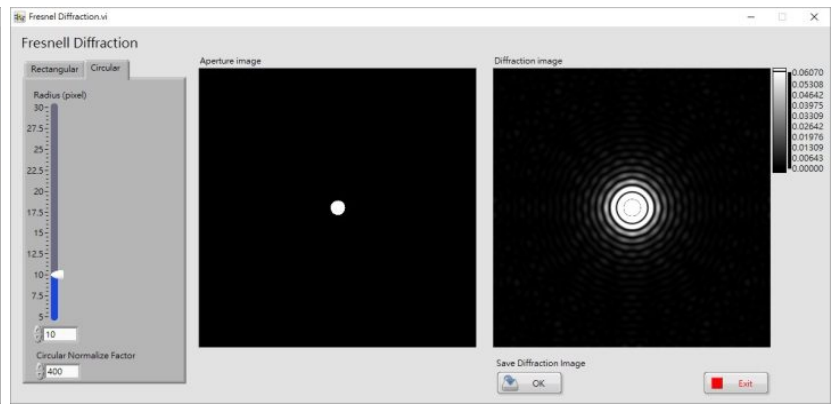

(b)

Figure 3 (a) Diffraction of square aperture (b) Diffraction of circular aperture http://dx.doi.org/10.1117/12.2257794.3

\subsection{Polarization wave and Poincare sphere}

Polarization is also abstract concept for many people. People know the electric field has the vector form [10], as eq (5).

$$
\left(\begin{array}{c}
E_{x} \\
E_{y}
\end{array}\right)=\left(\begin{array}{c}
A_{x} e^{i \delta} \\
A_{y}
\end{array}\right)
$$

Electric field $E_{x}$ and $E_{y}$ and their amplitude $A_{x}$ and $A_{y}$. There are phase retardation between $E_{x}$ and $E_{y}$ and the retardation value is $\delta$. The value $\operatorname{or}_{x}, E_{y}$ and $\delta$ can categorize these waves into linear polarization, circular polarization or elliptical polarization. The total electric wave is the combination of $\mathrm{x}$ and $\mathrm{y}$ direction field, and it is very difficult to draw a circular polarization in a blackboard. Student really needs some animation to help them construct this geometric relationship between the electric field in $\mathrm{x}, \mathrm{y}$ and the combination. Another idea also hard to realize is the Poincare sphere. Diffraction polarization state can also describe on the point of the sphere. The sphere is in Cartesian coordinates with three axes s1, s2, and s3. The positive s1 axis direction is horizontal polarization, and vertical polarization is in the opposite direction of $\mathrm{s} 1$ axis. The positive $\mathrm{s} 2$ axis direction is linear polarization in 45 degree, and the opposite direction is linear polarization in 135 degree. The north pole and south pole are left hand and right hand circular polarization. In eq (6), the inverse tangent of the amplitude ratio $A_{y}$ over $A_{x}$ makes $\psi$.

$$
\psi=\tan ^{-1}\left(\frac{A_{y}}{A_{x}}\right)
$$

The azimuthal angle is $2 \psi$ which describes the latitude where the point lies on the sphere and the retardation $\delta$ describe the angle between the point and S3 axis. With $2 \psi$ and retardation $\delta$, user can point out the polarizaiton state in Poincare sphere as eq. (7). The some complex idea in Poincare sphere is when the amplitude ratio is the same, and different retardation is rotate about $\mathrm{S} 1$ axis. Without computer visualization, it is not easy for student to understand.

$$
\left(\begin{array}{l}
s_{1} \\
s_{2} \\
s_{3}
\end{array}\right)=\left(\begin{array}{c}
\cos 2 \psi \\
\sin 2 \psi \cos \delta \\
\sin 2 \psi \sin \delta
\end{array}\right)
$$




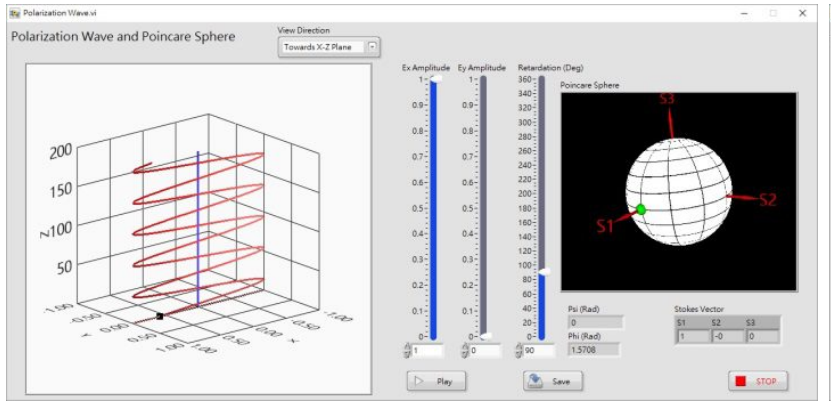

(a)

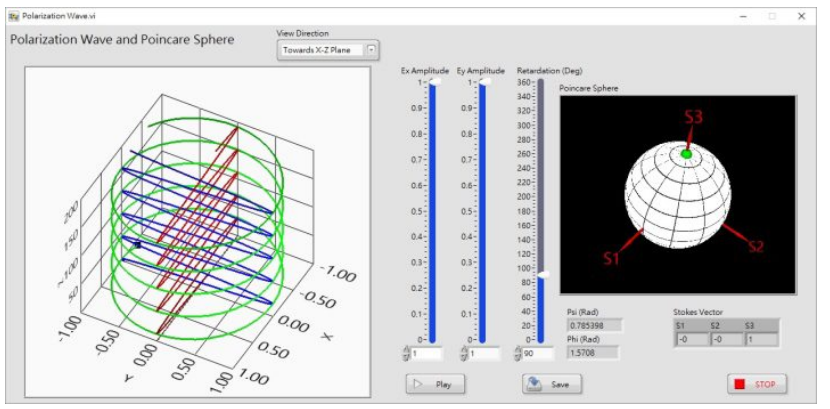

(b)

Figure 4 (a) Electric field only in $\mathrm{x}$ direction which form linear polarization. (b) Both $\mathrm{x}$ and $\mathrm{y}$ direction have linear polarization, and the retardation which between $\mathrm{x}$ and $\mathrm{y}$ is $\pi / 2$ which form circular polarization. http://dx.doi.org/10.1117/12.2257794.4

\subsection{Fabry-Perot etalon in spectrum domain}

Fabry-Perot etalon [12] is the cavity cause optical resonance. We meet it on the textbook, and almost every textbook draw the interference in frequency domain. However, in industrial instrument, engineer usually measure in spectrum domain not frequency domain. We can see the transmittance spectrum or reflectance spectrum. The Fabry-Perot etalon transmittance formula is shown in eq (8).

$$
\mathrm{T}_{\text {etalon }}=\frac{\left(1-\boldsymbol{R}_{\mathbf{1}}\right)\left(1-\boldsymbol{R}_{\mathbf{2}}\right)}{\left(1-\sqrt{\boldsymbol{R}_{\mathbf{1}}} \sqrt{\boldsymbol{R}_{\mathbf{2}}}\right)^{2}+4 \sqrt{\boldsymbol{R}_{\mathbf{1}} \boldsymbol{R}_{\mathbf{2}}} \sin ^{2}\left(\frac{2 \pi}{\lambda} L\right)}
$$

The $R_{1}$ and $R_{2}$ are reflectance in etalon surface 1 and surface 2 . $L$ and $\lambda$ are the length of the etalon and the wavelength of light. In the Figure 5(a), there are $1 \mathrm{~mm}$ etalon in mid-IR range. It is common when anti-reflection coating is good enough on LCOS-based WSS (wavelength selective switch). We can see the reflectance is $4 \%$ and induce the ripple in spectrum. In the Figure 5(b), we choose etalon near $3 \mu \mathrm{m}$ which is the typical cell gap for liquid crystal display. Some researcher even uses the spectrum to measure the empty cell gap [11]. In this program, center wavelength and reflectance are needed parameters. Another impartment parameter is the number of modes. It decides how many peaks shown in the spectrum.

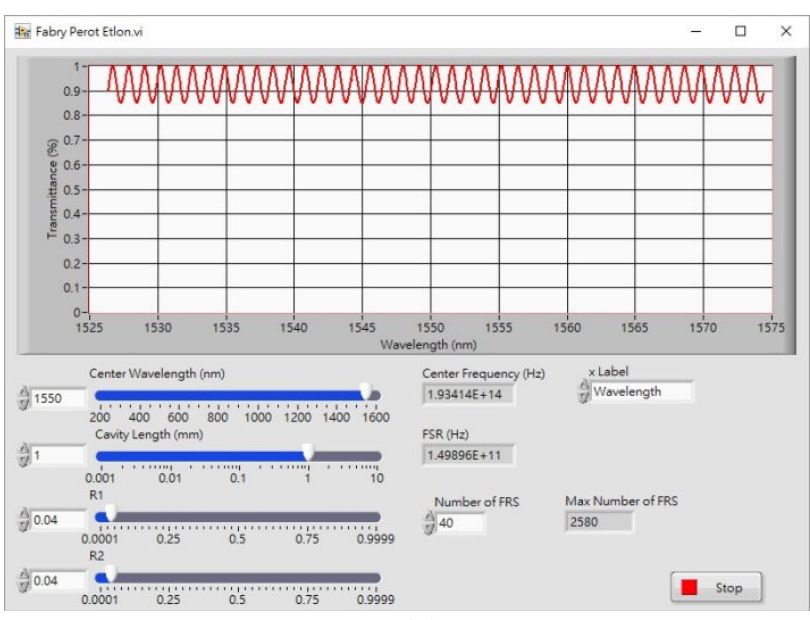

(a)

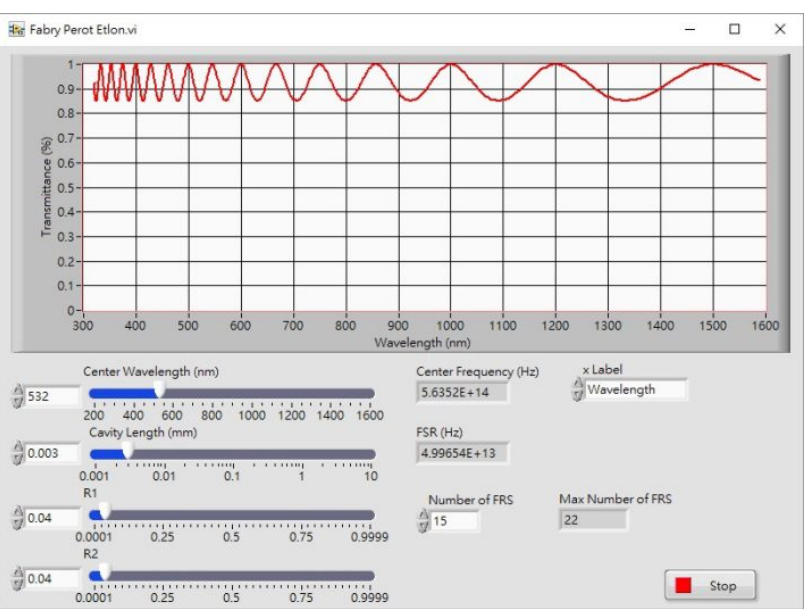

(b)

Figure 5 (a)Fabry-Perot interference for mid-IR light in 1mm thick etalon (b) Fabry-Perot interference for visible light in $3 \mu \mathrm{m}$ thick etalon. The longer wavelength has larger peak-to-peak spacing. http://dx.doi.org/10.1117/12.2257794.5 


\section{CONCLUSION}

In this paper, five photonics phenomenon had been discussed in software visualization and simulation with LabVIEW. It will be very helpful for user to learn a new idea with interactive software. The Snell's law simulation make user understand the relation among incident angle, refractive angle and refractive. The Hermite-Gaussian beam visualizes the complex mathematic formula of the electric distribution. The aperture diffraction can let user test different how different aperture size affect the diffraction pattern. The polarization wave visualization shows how electric wave look like in different amplitude and phase retardation and also connects its relation in Poincare sphere. The program points out where the polarization state lies on the Poincare sphere. The Fabry Perot etalon in wavelength spectrum demonstrate the ripple in spectrum in different.

Teachers can use software to enforce the insufficient in traditional lecture, and improve the interest of students. Students can learn through interactive software. They can also see difficult physical phenomenon in front of them. The biggest advantage of the software demonstration is the low cost, and can ensure that the phenomenon displayed properly in a short time. Experimental skill is very important, but in a limited educational environment, interactive software simulation may be a compromise way for learning these physical phenomena.

\section{REFERENCES}

[1] Alarcon, M., Lakhdar, Z. B., Culaba, I., Lahmar, S., Lakshminarayanan, V., Mazzolini, A., Niemela, J.“ Active learning in optics and photonics (ALOP): a model for teacher training and professional development" Optics Education and Outreach 7783, 778303 (2010)

[2] Diaz, A., Friedmana, J. S., Lucianoa, S., Martineza, S., Hernandez, A., de Jesus, J., Molina, N., Ramos, J. G., Casañas, A. and Maldonado, P. M. "Propagation of Electromagnetic Waves through Homogeneous Media" International Topical Meeting on Education \& Training in Optics an Photonics 9793, 97930I (2015)

[3] Ammar, A., Burman, R., Ghalila, H., Lakhdar, Z. B., Srinivasa, L., Varadharajan, Lahmar, S.and Lakshminarayanan, V. "Optics simulations with Python: Diffraction" International Topical Meeting on Education \& Training in Optics an Photonics 9793, 97930K (2015)

[4] Gerhard, C., Adams, G. "Easy-to-use software tools for teaching the basics, design and applications of optical components and systems" International Topical Meeting on Education \& Training in Optics an Photonics 9793, 97930N (2015)

[5] Wan, Y., Man, T., Tao, S.” Optics and Optics-based Technologies Education With the Benefit of LabVIEW" International Topical Meeting on Education \& Training in Optics an Photonics 9793, 979310 (2015)

[6] Marques, M. B., Rosa, C. C., Marques, P. V. S. "Eight year experience in open ended instrumentation laboratory" International Topical Meeting on Education \& Training in Optics an Photonics 9793, 979327 (2015)

[7] Wan, Y., Man, T., Tao, S. "Optics and Optics-based Technologies Education With the Benefit of LabVIEW" International Topical Meeting on Education \& Training in Optics an Photonics 9793, 97931O( 2015)

[8] Yariv, A. and Yeh, P. [Photonics: Optical Electronics in Modern Communicationsptical electronics in modern communications], Oxford University Press, 88-89 (2007).

[9] Goodman, J.W. [Fourier Optics], Roberts \& Compnay, 66-67 (2005)

[10] Yeh, P., Gu, C [Optics of Liquid Crystal Displays], 64-67 (2010)

[11] Yang, K. "Measurements of empty cell gap for liquid crystal displays using interferometric methods" Journal of applied physics, Journal of Applied Physics 64, 4780 (1988)

[12] Yariv, A. and Yeh, P. [Photonics: Optical Electronics in Modern Communicationsptical electronics in modern communications], Oxford University Press, 166-167 (2007). 